\title{
Ability to Recall Specific Detail and General Detail (Gist) in Young Old, Middle Old, and Older Adults
}

\author{
Victoria Alexander, Mark Bahr, Richard Hicks \\ School of Psychology, Bond University, Gold Coast, Australia \\ Email: vialexan@bond.edu.au
}

Received 21 October 2015; accepted 25 December 2015; published 28 December 2015

Copyright (c) 2015 by authors and Scientific Research Publishing Inc.

This work is licensed under the Creative Commons Attribution International License (CC BY). http://creativecommons.org/licenses/by/4.0/

\section{Open Access}

\begin{abstract}
Declining cognition has been associated with detrimental consequences such as decline in independence and reduced quality of life. If we can understand the nature of the decline, we may be able to reduce the detrimental consequences. It seems that with increasing age we remember the general detail of the stimuli, rather than the specific details. Recall of general information but failure to identify the specific instances previously known or studied is termed gist error, and this is seen to be indicative of age related change in memory. Previous studies have compared younger vs older age groups; meaning that the time course of these changes has not been established. We used three age groups in a trial move to examine whether age related change might start earlier than recognized from previous studies. Sixty-six participants aged 18 to 86 years completed computerised tasks assessing non-verbal and verbal gist recognition. Older adults recognised correctly fewer target stimuli than the young old and middle old cohorts on the non-verbal gist task. They also were significantly more likely to identify critical semantic lures related to the target ("general") than the other two age groups. In addition, on the verbal gist task, both middle and older adults recognised (incorrectly) more semantically related items than did the younger cohorts. These results are consistent and suggest that older adults are more likely to remember the general idea of stimuli (gist). However, older adults were able to recognize (recognized) more target stimuli than the young adults on the verbal task. This indicates probable dissociation between verbal and non-verbal representations and suggests cognitive decline is modular and related to specific functional decline, and is not generalized over all functions. The results also suggest that cognitive decline begins in early adulthood, rather than at the later spectrum of aging. These findings have implications for potential behavioural and pharmacological intervention.
\end{abstract}

\section{Keywords}

Aging, Age-Related Cognitive Decline, Gist, Memory 


\section{Introduction}

Declining cognition has been associated with a number of detrimental consequences for the aging individual, such as the inability to function independently. As older individuals are unable to look after their own needs, this may lead to institutionalisation. Institutionalised individuals are also likely to experience rapid cognitive deterioration and reduced quality of life (Formiga et al., 2009; St John, Montgomery, Kristjansson, \& McDowell, 2002). Pharmacological interventions (e.g., cholinesterase inhibitors), as well as behavioural interventions such as exercising and staying mentally active (e.g., doing crossword puzzles, reading books and newspapers and engaging in regular physical activity) may potentially slow the diseasing process (e.g., Boote, Lewin, Beverley, \& Bates, 2006; Cramer et al., 2012; Cruz-Oliver \& Morley, 2010; Small, Dixon, McArdle, \& Grimm, 2012; Wilson et al., 2002). Therefore, an understanding of the nature and onset of cognitive decline would be beneficial as both behavioural and pharmacological interventions could be implemented that improved quality of life and level of independence for the individual (e.g., Agrigoroaei \& Lachman, 2011; Luis, Loewenstein, Acevedo, Barker, \& Duara, 2003; Schmitter-Edgecombe \& Parsey, 2014).

There have been many studies on the physical and cognitive elements related to decline of memory in aging individuals, with some suggesting general overall decline occurs, while others suggest a modular of specific functional decline process. Several thrusts in the studies have included recent emphases, among others, on personality and cognitive decline (Luchetti, Terracciano, Stephan, \& Sutin, 2014); facial memory and emotion recognition (e.g., Alexander, Bahr, \& Hicks, 2014; Krendl, Rule, \& Ambady, 2014); and precursors to cognitive decline (e.g., Salthouse \& Soubelet, 2014). The current study examined whether memory over three older age groups was generalized or modular. Age related cognitive decline has been associated with a decline in memory for specific detail (Hudon et al., 2006). Decline in the ability to process the specific details of the object may result in remembering only the general, abstract detail or the "gist". Consequently, greater frequency of gist errors in aged populations might be indicative of changes in memory.

The Deese/Roediger-McDermott (DRM; Roediger \& McDermott, 1995) is a paradigm used that provides a measure of gist-based errors. In this paradigm, participants are required to study a list of semantically associated words (e.g., bed, rest, awake) during the learning phase. During the test phase, participants were presented words that were previously studied, unrelated but seen in the words studied and non-studied words along with a critical non-studied lure word which converged on the theme presented in the test phase (e.g., sleep). This parallels the prototypical member of the set. Roediger \& McDermott (1995) found that individuals were more likely to falsely recognise the non-studied critical lures than the unstudied unrelated lures (e.g., snow). A possible explanation is that after studying semantic associates, a gist representation of the list is encoded. Then by presenting related non-studied lures at test, this causes an illusionary feeling of familiarity that the word has been seen previously, increasing the likelihood of gist-based errors (Budson, Todman, \& Schachter, 2006). Alternatively, this may also suggest that people are likely to identify a stimulus which is similar to the typical members of the set.

Hudon et al. (2006), as part of their research, used the DRM (Roediger \& McDermott, 1995) with a sample of participants with mild cognitive impairment, Alzheimer's disease and older adult controls. Hudon et al. found all groups had similar levels of corrected true recognition of studied items when compared to the corrected recognition false recognition of related lures. Moreover, signal detection analysis comparing hits vs. critical lures found that sensitivity was at chance level for all groups. This suggested that all groups had evidence of intrusion errors in that they were unable to distinguish critical lures from the studied items. Hudon et al. (2006) acknowledge that the task may have been too demanding and consequently this result is likely to indicate floor effects in the data.

In the second experiment, a new sample of participants was administered a narrative task consisting of 128 high frequency and familiar words with 23 macropropositions and 24 micropropositions. During the study phase, participants were informed they had 3 minutes to read and memorize the content. Following the study phase, the text was hidden and participants were required to freely recall the study word-for-word. An immediate recall task was administered, followed by delayed recall task, which was performed 10 minutes later. Hudon et al. (2006) found that the Alzheimer's disease participants were the most impaired on both types of recall for both immediate and delayed conditions. The mild cognitive impairment participants were also significantly impaired on the text recall task, albeit not to the severity of the participants with Alzheimer's disease. An intriguing finding was that memory for micropropositions (detailed information) and macropropositions (gist) were impaired to the same degree for the participants with mild cognitive impairment and Alzheimer's disease. As expected, all 
groups including older adults had a higher proportion of recall for the macropropositions than micropropositions suggesting they were better at remembering the general idea over specific detail.

As there was only a significant difference between the mild cognitive impairment participants and older adults on text recall, this may suggest that memory decline is also dependent on the difficulty of the task. The text recall task requiring specific recall of propositions is likely to have required a higher level of cognitive resources (e.g., working memory and attention) than the DRM which requires the recognition of semantic associates (Roediger \& McDermott, 1995). This may indicate that working memory and attentional capacity are more impaired than semantic processing in early stages of cognitive decline. A limitation of Hudon et al.'s (2006) study was the lack of a relevant older age group that could have indicated whether older adults make more gist-based errors when compared to younger adults.

Using a different paradigm to the DRM, Guerin et al. (2012) implemented a visual memory task with a younger adult sample. In the first experiment, participants were shown coloured photographs divided into 4 conditions. In the baseline target condition, the target was shown along with two unrelated items. In the baseline foil condition, all 3 items were unrelated to the studied items. In the single related item condition one of the pictures was related to the study item, with the other 2 unrelated but no target picture was presented. In the target and related item condition, the target was presented as the adjacent item with the third item unrelated to the studied item. On each trial, participants were presented with three pictures. Two of the pictures were related to one another because they were exemplars of the same category and shared similar characteristics. In the first experiment Guerin et al. (2012) found that participants made more gist-based errors in the single related item condition. However, when the target was presented in the target and related condition there was a significant decrease in gist errors.

In the second experiment, participants were presented with a condition of two items that were related to the study item and a third item that was unrelated. Guerin et al. (2012) found that participants had no reduction in gist-based errors in the two related items condition. Similar to the first experiment, there was a significant reduction in gist errors in the target and related condition when participants had the benefit of the originally presented study item. The authors acknowledge that even though gist errors declined in the target and related conditions, they were not eliminated.

Guerin (2012) did not use an older age group and found their results for a younger age group: we speculated that if younger adults still make gist errors when provided target retrieval cues, then it is likely that older adults would also make gist errors in the presence of the target cue. In the current study, a pictures task was used where retrieval cues (target picture) were provided in each block. Based on initial pilot testing for the current study, it was found that younger adults still made gist errors in that they identified a similar item when the target was presented. Therefore, it was speculated that in an aging brain, older adults would be more likely to make gist errors on a picture recognition task even when retrieval cues were provided.

From the research, there is some evidence that older adults are more likely to remember the general idea 'the gist' rather than specific detail of stimuli. However, studies such as Guerin’s (2012) and Hudon et al.'s (2006) assessed either young or adults separately and did not compare between the two aged cohorts. Further, none of the articles identified used a middle old comparison group to assess whether decline for specific detail may occur earlier than "older adulthood". Contrary to earlier research that indicates cognitive decline occurs after the age of 65, there is evidence to suggest that it may begin as early as the second decade of life (e.g., Murre, Janssen, Rouw, \& Meeter, 2013).

To further investigate whether age related decline is reflected by decline for specific detail, the current study investigated whether older people are more likely to make gist errors than the younger cohort. If older adults are more likely to remember only the general idea or gist, this may provide evidence that there is some level of cognitive decline. From the literature (e.g., Hudon et al., 2006), there was some indication that older adults are better at remembering the general idea over specific detail. Therefore, it was predicted that older adults were more likely to make gist errors than younger adults.

An additional aim was to assess whether age related decline is modular or generalised. If there is decline for specific detail but memory for the general idea remains intact, this could indicate that memory subsystems are differentially affected or decline independently. This could suggest that there are separate stores for "gist" and "item specific memory.

As previously mentioned, memory for the general idea might be indicative of storing generally. Therefore, if decline for memory of specific material is observed but that memory for the general idea specific remains intact, 
this could indicate generalised decline. This generalised decline may be caused from a variety of sources such as an accumulation of amyloid $\beta$, which has been associated with memory loss (Lim, Lee, Barton, \& Moon, 2011).

However, if decline for verbal material is observed but non-verbal remains intact, this could be indicative of dissociation of verbal and non-verbal representations and provide support for modular decline with increasing age.

\section{Method}

\subsection{Participants}

A sample of 70 participants was recruited from South-Eastern Queensland to participate in the study. The young adults sample was comprised of first year students from a university who received course credit for participation in the research. The other participants were from the local community in South-Eastern Queensland. Screening of the data leads to the final sample total of 66, comprising of 49 females $(74.2 \%)$ and 17 males (25.8\%). The age of the entire sample ranged from 18 to 86 years $(M=50.06, S D=21.52)$. For highest education obtained, 42 (63.6\%) participants nominated high school, 17 (25.8\%) university, 5 (7.6\%) nominated T.A.F.E/college and 2 nominated primary school (3.0\%). From the participants, 35 (53.0\%) were currently taking medication for illnesses e.g., high blood pressure, high cholesterol, diabetes, vascular problems. The other 31 participants (47.0\%) were not currently taking medication.

\subsection{Instruments}

\subsubsection{Non-Verbal Gist}

A computerised picture recognition task was used as a measure of non-verbal gist. The stimuli for the initial learning phase consisted of pictorial exemplars from 27 categories (e.g., a robin was an exemplar for the bird category). The categories consisted of balls, teddy bears, beds, birds, butterflies, cars, cats, chairs, clocks, dogs, fans, fish, picture frames, eyeglasses, grapes, houses, insects, pens, shoes, socks, staplers, teapots, tissue boxes, tomatoes, trees, t-shirts and umbrellas.

In the learning phase, each of the 27 exemplars was presented individually on the screen. The exemplars were presented for $2000 \mathrm{~ms}$ to ensure sufficient encoding of each picture. After each stimulus had been shown on the screen, a 100 ms mask and 500 ms blank time were provided to allow the participant to reset prior to the presentation of the next exemplar.

A number search task was presented prior to the experimental phase. In the number search task, participants were presented blocks of numbers and were required to count the number of $7 \mathrm{~s}$. This was designed to provide a longer time period between learning and experimental phases and make the task more difficult.

In the experimental phase, participants were presented 27 trials. On each trial, 4 pictures were presented. One of the items was the initial exemplar from the learning phase. The second item was close to the exemplar in semantic similarity, usually in colour, or another type of the object (e.g., a different type of clock). The third item was functionally related to the original item (e.g., an hour-glass similar to a clock but still telling time). The final item was unrelated (e.g., a vase). Participants were required to select the item that was presented in the learning phase. The task was self-paced.

\subsubsection{Verbal Gist}

A computerized variation of the Deese/Roediger-McDermott (DRM; Roediger \& McDermott, 1995) was used as a measure of non-verbal gist. The words used in this task were also adapted from Roediger and McDermott, as they had been found to be capable of detecting gist errors in a younger cohort.

In the learning phase, 12 words were presented individually on the screen. The words were also read aloud by the program voice-over. Six of the words presented were related to the non-studied critical lure and six were unrelated. Each of the words was presented for $2000 \mathrm{~ms}$. A blank time of $500 \mathrm{~ms}$ between each presentation was provided to allow the participant to reset prior to the next word.

In the experimental phase participants were presented with 4 blocks of 19 words (the unstudied critical lure, 6 studied words related to the lure, 6 related unstudied words and 6 unrelated studied words taken from another category). The participants were required to identify whether the word was an "old" word (seen in the study phase) or a "new" word (not seen in the study phase). They were then required to select the level of confidence 
they had in their decision with either "know" (confident they saw the word) or "remember" (think they saw the word were not entirely sure). Once $20000 \mathrm{~ms}$ had been reached, a timeout would occur and the next word in the block would be presented.

\subsection{Procedure}

The study was conducted in accordance with the Australian National Statement on Ethical conduct in Human Research (2007), prior to commencement of the study the research protocol was approved by the Bond University Human Research Ethics committee (BUHREC). Prior to administration, the tasks were counterbalanced to reduce the potential of order effects. Participants were verbally instructed to read the instructions on the laptop screen and indicate that they understood prior to the commencement of each task.

\subsection{Design}

As a guide to decline over time, we decided to examine three main age groups rather than just two as has been the case in much earlier research. If the decline were evident across the three groups then this would be an indication that further study should follow with larger samples across the age ranges. Our interest in verbal versus non-verbal decline was examined separately in the experimental design described above (under instruments), and was aimed at gathering further evidence to indicate whether cognitive decline operated differently for different functions or was general over all functions.

\subsection{Statistical Analysis}

Multivariate Analysis of Variance (MANOVA) was used to assess the effect of Age (Young Old (18 - 49), Middle Old (50 - 64), and Older Adults (65+)) on the dependent variables of Recognition and Latency (Non-Verbal Gist) and Proportion of Error (Verbal Gist).

\subsubsection{Non-Verbal Gist Study}

The independent variable was Age (Young (18 - 49), Middle Old (50 - 64), and Older Adults (65 and above)). The dependent variables were Recognition to Identify Pictorial Stimuli (Target, Semantically Related, Functionally Related, Unrelated) and Latency to Identify Pictorial Stimuli (Target, Semantically Related, Functionally Related, Unrelated).

\subsubsection{Verbal Gist Study}

The independent variable was Age (Young (18 - 49), Middle Old (50 - 64), and Older Adults (65 and above)). The dependent variable was Proportion of Error (Target, Critical Lures, Semantically Related, and Unrelated).

\section{Results}

Initial data checking using IBM SPSS Statistics 21 confirmed that the data met the assumptions for Multivariate analysis of variance (MANOVA). As Box's M was violated, a more conservative Pillai's criterion was used to interpret multivariate significance unless otherwise indicated.

\subsection{Non-Verbal Gist}

A ONEWAY between groups MANOVA (Multivariate Analysis of Variance) was conducted to assess the effect of Age (Young Old (18 - 49), Middle Old (50 - 64), and Older Adults (65+)) on Recognition to Identify Pictorial Stimuli (Target, Semantically Related, Functionally Related, Unrelated). $\alpha$ was set at .05 apriori. There was a significant effect of Age on Recognition to Identify Pictorial Stimuli $\left(F(8,120)=2.06, p=.045\right.$, partial $\eta^{2}=.12$, power $=.81$ ). As the overall test of the weighted linear composite was significant, each of the dependent variables was then considered separately. Table 1 shows the results.

Analysis of the dependent variables separately showed that there was a significant effect of Age on Recognition of Target Pictorial Stimuli, with older adults recognising fewer target stimuli than young and middle old adults (Table $1, F(2,63)=7.30, p=.001$, partial $\eta^{2}=.19$, power $\left.=.93\right)$. 
Table 1. Mean proportion recognition and standard deviations of pictorial stimuli by age group: for young old, middle old, and older adults.

\begin{tabular}{cccc}
\hline Types of Recognition & \multicolumn{3}{c}{ Age Group } \\
\cline { 2 - 4 } & $\begin{array}{c}\text { Young Old } \\
\text { M (sd) }\end{array}$ & $\begin{array}{c}\text { Middle Old } \\
\text { M (sd) }\end{array}$ & $\begin{array}{c}\text { Older Adults } \\
\text { M (sd) }\end{array}$ \\
\hline Target & $.94^{\mathrm{a}}(.10)$ & $.96^{\mathrm{b}}(.04)$ & $.87(.09)$ \\
Semantically Related & $.04^{\mathrm{a}}(.06)$ & $.02^{\mathrm{b}}(.03)$ & $.09(.07)$ \\
Functionally Related & $.02(.04)$ & $.01(.02)$ & $.03(.04)$ \\
Unrelated & $.00(.01)$ & $.00(.01)$ & $.01(.02)$ \\
\hline
\end{tabular}

NB: aDifference between young and older adults; ${ }^{\mathrm{b}}$ Difference between young and middle old adults.

To investigate group differences between Age, a series of post hoc analyses were conducted using Tukey's $H S D$ with $\alpha=.05$. There was a significant difference on recognition of the target picture between young and older adults, with older adults recognising fewer target stimuli than young adults (refer to Table 1). There was also a significant difference between middle old and older adults, with older adults recognising fewer target stimuli than the middle old adults. However, there was no significant difference between young and middle old adults.

Older adults recognized significantly more semantically related stimuli than young and middle old adults $(F(2$, $63)=7.72, p=.001$, partial $\eta^{2}=.20$, power $=.94$ ). Post hoc analyses were conducted using Tukey's HSD with $\alpha$ $=.05$. There was a significant difference between young and older adults, with older adults recognising more semantically related stimuli than young adults. There was also a significant difference between middle and older adults, with older adults recognising more semantically related stimuli than middle old adults. However, there was no significant difference between young and middle old adults recognition of targets. There was no significant effect of Age on Recognition of Functionally Related Pictorial Stimuli $\left(F(2,63)=1.93, p=.15\right.$, partial $\eta^{2}$ $=.06$, power $=.39)$ or Unrelated Pictorial Stimuli $\left(F(2,63)=.84, p=.44\right.$, partial $\eta^{2}=.03$, power $\left.=.19\right)$.

As can be seen from Table 1, older adults recognised significantly fewer target stimuli than the young and middle old adults. They also were significantly more likely to identify critical semantic lures related to the target than the other two age groups. This is consistent with the gist hypothesis that older adults' recognition performance is more influenced by general characteristics of the stimuli (category of stimulus) than younger participants.

\section{Latency}

A ONEWAY between groups MANOVA assessed the effect of Age (Young Old (18 - 49), Middle Old (50 - 64), and Older Adults (65+)) on Latency to Identify Pictorial Stimuli (Target, Semantically Related, Functionally Related, Unrelated). There was a significant effect of Age on Latency $\left(F(8,122)=4.00, p<.001\right.$, partial $\eta^{2}$ $=.20$, power $=.99$ ).

Univariate analysis of the dependent variables separately, indicated a significant effect of Age on Latency to Recognise Target Pictorial Stimuli, with older adults taking longer to recognise targets than young and middle old adults $\left(F(2,63)=10.09, p<.001\right.$, partial $\eta^{2}=.24$, power $\left.=.98\right)$. Tukey's $H S D(\alpha=.05)$ revealed a significant difference in target latency with older adults taking longer to recognise target stimuli than young adults (refer to Table 2). Middle old adults took significantly longer to recognise targets than young adults. However, there was no significant difference between middle and older adults.

There was also a significant effect of Age on Latency to Recognise Semantically Related Pictorial Stimuli, $\left(F(2,63)=9.00, p<.001\right.$, partial $\eta^{2}=.22$, power $\left.=.97\right)$. Post hoc analyses using Tukey's HSD $(\alpha=.05)$ indicated older adults had significantly longer latencies for semantically related pictorial stimuli than young adults (as indicated in Table 2). Older adults also had significantly longer latencies for semantically related pictorial stimuli than middle old adults. However, there was no significant difference between young and middle old adults latencies (refer to Table 2).

There was no significant effect of Age on Latency to Recognise Functionally Related Pictorial Stimuli (F(2, $63)=.46, p=.633$, partial $\eta^{2}=.01$, power $\left.=.12\right)$ or Latency to Recognise Unrelated Pictorial Stimuli $(F(2,63)$ $=.34, p=.711$, partial $\eta^{2}=.01$, power $\left.=.10\right)($ refer to Table 2$)$. 
Table 2. Mean latency (ms) to recognise pictorial stimuli by age group: young old, middle old, and older adults.

\begin{tabular}{cccc}
\hline Types of Recognition & \multicolumn{3}{c}{ Age Group } \\
\cline { 2 - 4 } & $\begin{array}{c}\text { Young Old } \\
\text { M (sd) }\end{array}$ & $\begin{array}{c}\text { Middle Old } \\
\text { M (sd) }\end{array}$ & $\begin{array}{c}\text { Older Adults } \\
\text { M (sd) }\end{array}$ \\
\hline Target & $1889^{\text {ab }}(457)$ & $2397(644)$ & $2769(855)$ \\
Semantically Related & $145^{\mathrm{a}}(222)$ & $100^{\mathrm{c}}(188)$ & $440(400)$ \\
Functionally Related & $61(104)$ & $79(187)$ & $102(143)$ \\
Unrelated & $33(150)$ & $12(36)$ & $36(84)$ \\
\hline
\end{tabular}

NB: ${ }^{\mathrm{a}}$ Difference between young and older adults; ${ }^{\mathrm{b}}$ Difference between young and middle old adults; ${ }^{\mathrm{c}}$ Difference between middle and older adults.

\subsection{Verbal Gist}

A ONEWAY between groups MANOVA (Multivariate Analysis of Variance was conducted to assess the effect of Age (Young Old (18 - 49), Middle Old (50 - 64) and Older Adults (65+)) on Proportion of Error (Target, Critical Lures, Related and Unrelated). There was a significant effect of Age on Proportion of Error $(F(4,126)=$ $6.15, p<.001$, partial $\eta^{2}=.16$, power $=.99$ ). As the overall test of the weighted linear composite was significant, each of the dependent variables was then considered separately.

Analysis of the dependent variables separately showed that there was a significant effect of Age on Proportion of Error for Target Recognition, with younger adults having a higher proportion of error in recognising target stimuli than middle and older adults $\left(F(2,63)=10.53, p<.001\right.$, partial $\eta^{2}=.25$, power $=.99$ ). An unexpected finding was although there was a significant difference between the young and old adults; the young adults had a higher proportion of error in recognising target stimuli than older adults (refer to Table 3). There was a significant difference between the young and middle old adults, but again young adults had a higher proportion of error in recognising target stimuli than middle old adults (refer to Table 3). However, there was no significant difference between middle and older adults (refer to Table 3).

There was a significant effect of Age on Proportion of Error for Semantically Related Item Recognition, with middle and older adults having a higher proportion of error in recognising semantically related stimuli than young adults $\left(F(2,63)=14.96, p<.001\right.$, partial $\eta^{2}=.32$, power approaching 1$)$. To investigate group differences between Age, a series of post hoc analyses were conducted using Tukey's HSD with $\alpha=.05$. There was a significant difference between younger and older adults, with older adults having a higher proportion of error in recognising semantically related stimuli than young adults. There was also a significant difference between younger and middle old adults, with middle old adults having a higher proportion of error in recognising semantically related stimuli than young adults. However, there was no significant difference between the middle and older adults (refer to Table 3).

Again, it was found that older adults recognised stimuli that were semantically related to the target. It was also found that middle old adults recognised stimuli that were semantically related. This again suggests older adults are making gist errors. An intriguing finding was that younger adults made significantly more errors in recognition of the target stimuli than both the young and middle old adults.

\section{Discussion}

From the literature (e.g., Hudon et al., 2006), there was some indication that older adults are better at remembering the general idea over specific detail. If older adults are more likely to remember the general idea or gist, this may provide evidence that there is some level of cognitive decline. Therefore, it was predicted that older adults were more likely to make gist errors than younger adults.

\subsection{Non-Verbal Gist}

As predicted, older adults were less able to accurately recognise the target on the pictorial task than the younger and middle older adults. This might suggest that older adults were unable to encode a specific episodic trace of the target picture. For example, a target of a specific umbrella is encoded as distinct from a generic representation of an umbrella, or something you use to keep dry (which may elicit a macintosh). It is thought that a specific episodic or item specific trace is more likely to be encoded when the trace is distinctive and a new association 
Table 3. Mean proportion of error and standard deviations for recognition of verbal stimuli by age group: young old, middle old, and older adults.

\begin{tabular}{cccc}
\hline Types of Recognition & \multicolumn{3}{c}{ Age Group } \\
\cline { 2 - 4 } & $\begin{array}{c}\text { Young Old } \\
\text { Target }\end{array}$ & $\begin{array}{c}\text { Middle Old } \\
\text { M (sd) }\end{array}$ & $\begin{array}{c}\text { Older Adults } \\
\text { M (sd) }\end{array}$ \\
Semantically Related & $.47^{\mathrm{ab}}(.21)$ & $.19(.17)$ & $.27(.28)$ \\
$.86(.23)$ \\
\hline
\end{tabular}

NB: ${ }^{\mathrm{a} D i f f e r e n c e ~ b e t w e e n ~ y o u n g ~ a n d ~ o l d e r ~ a d u l t s ; ~}{ }^{\mathrm{b}}$ Difference between young and middle old adults.

is stored over a new set of units. However, the pictures presented in the task were of common everyday items e.g., lamps, dogs and cats which are unlikely to be distinctive to the older adults and therefore may not have needed a high level of processing to identify the item.

In addition, it was found that older adults recognised more of the semantically related items (e.g., recognising the red shirt when the black shirt was the target) than the younger and middle old adults. The finding that older adults were less accurate in recognising the item specific target and more likely to identify the item that is semantically similar supports the argument that older adults are storing generally rather than as a unique trace.

A puzzling finding was that younger adults were less accurate in recognising the target and made more semantically related errors than the middle adults, albeit not significantly. This could suggest that the younger participants had some level of difficulty during the initial study phase and therefore only encoded a general representation of the item, rather than the specific details. This finding provides partial support for Guerin et al.'s (2012) research that found younger adults still made gist errors in the presence of the target.

However, it was found that there was no significant difference between the three cohorts on functionally related and unrelated related items. This suggests that the older adults did not make gross semantic confusions, which might suggest some preservation of specificity of encoding. It is also possible that differences on functionally and unrelated items may have been detected in more cognitively impaired individuals.

A potential limitation of the task was the differentiation between semantically, functional and unrelated item in that some of the unrelated items could be classified as related and thus still had some relatedness to the target item. For example, one could argue that some of the unrelated items could still be classified as being somewhat related to the task (e.g., a hockey stick is still related to a ball because it is used for sporting purposes). In addition, some of the functionally related may also have been classified as semantically related (e.g., for the glasses category a pair of sunglasses was used as a functionally related item). Since the main focus was to assess whether older adults are more likely to make gist errors, future research may consider eliminating the functionally related and unrelated category and implementing more semantically related items. However, despite the lack of a distinct differentiation between items, it was still found that older adults consistently selected the item that would be considered most semantically related to the target.

An analysis of response latencies indicated that older adults were significantly slower in the recognition of both target and semantically related pictorial stimuli than young and middle old adults. It was also identified that middle old adults were significantly slower than the young adults in recognising target pictorial stimuli. As there was some evidence of a decline in accuracy, this suggests a generalised slowing of the ageing brain. One account of generalized slowing attributes slowing to the physiological consequences of an increase in amyloid $\beta$ plaque accretion in the ageing brain. Amyloid $\beta$ build-up has been associated with disturbances of neural network activity or neuronal death (Cramer et al., 2012; Palop \& Mucke, 2010). Although there is still some debate as to the exact role of the plaque buildups, recent research suggests that the rate of deposition of amyloid $\beta$ is similar in neurotypical and sufferers of Alzheimer's disease but that the clearance of amyloid is impaired in Alzheimer's sufferers. There is also some evidence that this may begin to occur in middle adulthood.

There were no significant differences between the three aged cohorts in latencies for functional and unrelated category responses. However, this is likely to be as a result of older adults' identifying more semantically and target items over functionally related and unrelated items. As mentioned previously, it is likely that older adults still retain some level of detail of stimuli and therefore are unlikely to make these types of errors. Again, it is possible that cognitively impaired individuals would have identified more functional and unrelated items. 


\subsection{Verbal Gist}

A puzzling finding was that younger adults made significantly more errors in recognition of targets on the verbal task than the middle and older adults. One would expect that younger adults should be able to form an item specific representation. However, this does not seem to be the case. One possibility is that the sample inadvertently contained younger adults with undiagnosed impairments. More likely, it could indicate a task demand characteristic in that the younger adults found the task too easy or less engaging. It is likely that concerns about memory would be greater in older adults and this may motivate them to engage with the task at a higher level.

As predicted, both middle and older adults made significantly more semantically related errors. The fact that these cohorts identified more semantically related items suggest that they are still likely to recognise an item that is semantically similar to the target. Moreover, the finding that middle old adults were more likely to select semantically related items suggests that storing generally may begin to occur in middle adulthood.

A possible limitation was the lack of practice trials given prior to commencing the test phase. Future research could consider incorporating practice trials prior to the commencement of the test phases to ensure that participants have an adequate understanding of the task. Another potential issue was that participants were only provided one trial of each set of words. Budson et al. (2000) found that recognition increased after repeated presentations of the trials, which may suggest that several repetitions of the task could increase memory performance. However, it could be argued that the repeating presentations of stimuli could cause induce potential artifacts and increase the likelihood for intrusion errors.

\subsection{Age-Related Cognitive Decline-Is Decline Generalised or Modular?}

A further aim of the current study was to gather evidence towards assessing whether age related decline is modular or generalised. On the non-verbal gist task, there was evidence of age related decline for recognition of target and increased tendency to make gist errors. This provides some evidence of generalised decline in the older adults. Although older adults also made gist errors on the verbal gist task, they were also able to recognise target stimuli. Although it is not entirely clear, there is some indication of dissociation between verbal and non-verbal representations. This provides support for modular decline with increasing age and suggests that decline may occur differentially throughout the brain.

Although older adults had higher recognition of the target on the verbal gist task than the younger adults, this could be indicative of performance decrement in the younger adults rather than lack of decline in the older sample. There was also some indication that remembering the general detail of stimuli may occur earlier as middle old adults also had a tendency to make gist errors on the verbal gist task.

\subsection{Limitations of Study and Directions for Future Research}

As mentioned previously, a potential limitation of the non-verbal gist task was the differentiation between semantically, functional and unrelated item in that some of the unrelated items could be classified as related and thus still had some relatedness to the target item. For example, one could argue that some of the unrelated items could still be classified as being somewhat related to the task (e.g., a hockey stick is still related to a ball because it is used for sporting purposes). In addition, some of the functionally related may also have been classified as semantically related (e.g., for the glasses category a pair of sunglasses was used as a functionally related item). Since the main focus was to assess whether older adults are more likely to make gist errors, future research may consider eliminating the functionally related and unrelated category and implementing more semantically related items.

A possible limitation of the verbal gist task was the lack of practice trials given prior to commencing the test phase. Future research could consider incorporating practice trials prior to the commencement of the test phases to ensure that participants have an adequate understanding of the task. There is also some indication of sampling bias in the young adult cohort. Future research could consider resampling to see whether the same results on the verbal gist task (young adults recognizing fewer targets than middle and older adults) are still obtained.

\subsection{Conclusion}

The results of the study suggest that cognitive decline occurs asynchronously, which means that it is modular, not generalised. The results also suggest that the disease process may begin in early adulthood, rather than at the later spectrum of aging. This has implications for potential pharmacological and behavioural interventions that may help to preserve cognitive function and improve quality of life. 


\section{References}

Agrigoroaei, S., \& Lachman, M. E. (2011). Cognitive Functioning in Mid-Life and Old Age: Combined Effects of Psychosocial and Behavioural Factors. The Journals of Gerontology B: Psychological Sciences and Social Sciences, 668, 130-140. http://dx.doi.org/10.1093/geronb/gbr017

Alexander, V., Bahr, M., \& Hicks, R. (2014). Emotion Recognition and Verbal and Non-Verbal Memory Changes among Older Adults: Is Decline Generalised or Modular? GSTF Journal of Psychology, 1, 14-21.

Boote, J., Lewin, V., Beverley, C., \& Bates, J. (2006). Psychosocial Interventions for People with Moderate to Severe Dementia: A Systematic Review. Clinical Effectiveness in Nursing, 9, 1-15. http://dx.doi.org/10.1016/j.cein.2006.06.002

Budson, A. E., Daffner, K. R., Desikan, R., \& Schachter, D. L. (2000). When False Recognition is Unopposed by True Recognition: Gist-Based Memory Distorting in Alzheimer’s Disease. Neuropsychology, 14, 277-287.

Budson, A. E., Todman, R. W., \& Schacter, D. L. (2006). Gist Memory in Alzheimer’s Disease: Evidence from Categorised Pictures. Neuropsychology, 20, 113-122.

Cramer, P. E., Cirrito, J. R., Wesson, D. W., Lee, D. C. Y., Karlo, C. J., Zinn, A. E., Casali, B. T., James, M. J. et al. (2012). ApoE-Directed Therapeutics Rapidly Clear $\beta$-Amyloid and Reserves Deficits in AD Mouse Models. Science, 335, 15031506.

Cruz-Oliver, D. M., \& Morley, J. E. (2010). Early Detection of Cognitive Impairment: Do Screening Tests Help? American Directors Association, 1-10. http://dx.doi.org/10.1016/j.jamda.2009.10.012

Formiga, F., Fort, I., Robles, M. J., Riu, S., Sabartes, O., Barranco, E., Catena, J. et al. (2009). Comorbidity and Clinical Features in Elderly Patients with Dementia: Differences According to Dementia Severity. The Journal of Nutrition, Health \& Aging, 13, 423-427. http://dx.doi.org/10.1007/s12603-009-0078-x

Guerin, S. A., Robbins, C. A., Gilmore, A. W., \& Schacter, D. L. (2012). Retrieval Failure Contributes to Gist-Based False Recognition. Journal of Memory and Language, 66, 68-78. http://dx.doi.org/10.1016/j.jml.2011.07.002

Hudon, C., Belleville, S., Souchay, C., Gely-Nargeot, M. C., Chertkow, H., \& Gauthier, S. (2006). Memory for Gist and Detail Information in Alzheimer's Disease and Mild Cognitive Impairment. Neuropsychology, 20, 566-577. http://dx.doi.org/10.1037/0894-4105.20.5.566

Krendl, A. C., Rule, N. O., \& Ambady, N. (2014). Does Aging Impair First Impression Accuracy? Differentiating Emotion Recognition from Complex Social Inferences. Psychology \& Aging, 29, 482-490. http://dx.doi.org/10.1037/a0037146

Lim, T. S., Lee, H. Y., Barton, J. J. S., \& Moon, S. Y. (2011). Deficits in Face Perception in the Amnestic Form of Mild Cognitive Impairment. Journal of the Neurological Sciences, 309, 123-127. http://dx.doi.org/10.1016/j.jns.2011.07.001

Luchetti, M., Terracciano, A., Stephan, Y., \& Sutin, A. R. (2014). Personality and Cognitive Decline in Older Adults: Data from a Longitudinal Sample and Meta-Analysis. The Journals of Gerontology B: Psychological Sciences and Social Sciences (Online).

Luis, C. A., Loewenstein, D. A., Acevedo, A., Barker, W. W., \& Duara, R. (2003). Mild Cognitive Impairment: Directions for Future Research. Neurology, 61, 438-444. http://dx.doi.org/10.1212/01.WNL.0000080366.90234.7F

Murre, J. M. J., Janssen, S. M. J., Rouw, R., \& Meeter, M. (2013). The Rise and Fall of Immediate Delayed Memory for Verbal and Visuospatial Information from Late Childhood to Late Adulthood. Acta Psychologica, 142, 96-107. http://dx.doi.org/10.1016/j.actpsy.2012.10.005

Palop, J. J., \& Mucke, L. (2010). Amyloid- $\beta$-Induced Neuronal Dysfunction in Alzheimer’s Disease: From Synapses toward Neural Networks. Nature Neuroscience, 13, 812-818. http://dx.doi.org/10.1038/nn.2583

Roediger, H. L., \& McDermott, K. B. (1995). Creating False Memories: Remembering Words Not Presented in Lists. Journal of Experimental Psychology: Learning, Memory and Cognition, 21, 803-814. http://dx.doi.org/10.1037/0278-7393.21.4.803

Salthouse, T. A., \& Soubelet, A. (2014). Hetrogenous Ability Profiles May Be a Unique Indicator of Impending Cognitive Decline. Neuropsychology, 28, 812-818. http://dx.doi.org/10.1037/neu0000100

Schmitter-Edgecombe, M., \& Parsey, C. (2014). Assessment of Functional Change Cognitive Correlates in the Progression from Healthy Cognitive Aging to Dementia. Neuropsychology, 286, 881-893. http://dx.doi.org/10.1037/neu0000109

Small, B. J., Dixon, R. A., McArdle, J. J., \& Grimm, K. J. (2012). Do Changes in Lifestyle Engagement Moderate Cognitive Decline in Normal Aging? Evidence from the Victoria Longitudinal Study. Neuropsychology, 26, 144-155. http://dx.doi.org/10.1037/a0026579

St John, P. D., Montgomery, P. R., Kristjansson, B., \& McDowell, I. (2002). Cognitive Scores, Even within Normal Range, Predict Death and Institutionalization. Age and Aging, 31, 373-378. http://dx.doi.org/10.1093/ageing/31.5.373 